in vivo $36: 121-131(2022)$

doi:10.21873/invivo.12683

\title{
Long Non-coding RNA LOC285194 Promotes Epithelial Ovarian Cancer Progression via the Apoptosis Signaling Pathway
}

\author{
GA WON YIM ${ }^{1}$, DAE WOO LEE ${ }^{2}$, JAE IN KIM ${ }^{3}$ and YOUNG TAE KIM ${ }^{3}$ \\ ${ }^{1}$ Department of Obstetrics and Gynecology, \\ Dongguk University College of Medicine, Goyang, Republic of Korea; \\ ${ }^{2}$ Department of Obstetrics and Gynecology, Bucheon St. Mary Hospital, \\ Catholic University Medical College, Bucheon, Republic of Korea; \\ ${ }^{3}$ Institute of Women's Life Medical Science, Department of Obstetrics and Gynecology, \\ Yonsei University College of Medicine, Seoul, Republic of Korea
}

\begin{abstract}
Background/Aim: To explore the molecular mechanism and clinical significance of a newly identified IncRNA LOC285194 in epithelial ovarian cancer (EOC). Materials and Methods: LOC285194 transcript levels were analyzed in EOC cells compared to normal cells. Small interfering RNAs were used to suppress LOC285194 expression. Levels of apoptosis-related proteins were determined by western blot. LOC285194 expression in ovarian cancer and non-tumor tissues were compared with clinicopathologic and survival data. Results: Knockdown of LOC285194 decreased cell migration and proliferation, enhanced reactive oxygen species production and resulted in increased levels of proteins of the extrinsic apoptotic signaling pathway. LOC285194 expression level was higher in ovarian cancer tissues compared to control. Overall survival was significantly shorter in patients with high LOC285194 expression. Lymph node metastasis and high LOC285194 expression were significant prognostic factors of mortality $(H R=4.614$ and $5.880 ; p=0.026$ and $p=0.002$, respectively). Conclusion: LOC285194 can promote the progression of EOC via an anti-apoptotic mechanism. It may serve as a novel biomarker for predicting prognosis of EOC.
\end{abstract}

This article is freely accessible online.

Correspondence to: Young Tae Kim, MD, Ph.D., Institute of Women's Life Medical Science, Division of Gynecologic Oncology, Department of Obstetrics and Gynecology, Yonsei University College of Medicine, 50 Yonsei-ro, Seodaemun-gu, Seoul 03722, Republic of Korea. Tel: +82 222282240, Fax: +82 23138357, e-mail: ytkchoi@yuhs.ac

Key Words: Epithelial ovarian cancer, lncRNA, LOC285194, apoptosis.
Long non-coding RNAs (lncRNAs) are non-protein coding RNAs longer than 200 bases in length that may function as oncogenes or tumor suppressors, similar to protein-coding genes. Increasing evidence suggests that lncRNAs play a critical role in cellular regulation during various biological processes such as cell cycle progression, apoptosis, cell differentiation, and proliferation (1-3). LncRNAs are known to act as both positive and negative regulators of apoptosis in different tumor types, such as AFAP1-AS1 in esophageal adenocarcinoma and GAS5 in prostate cancer (4-7). The expression of many lncRNAs is associated with ovarian cancer as well, namely MALAT1, HOTAIR, H19, XIST, and 151 others. As in other cancers, these lncRNAs play a major role in ovarian cancer cell proliferation, apoptosis, the cell cycle, migration, invasion, metastasis, and drug-resistance. To date, a total of 15,767 annotated lncRNAs have been identified in the GENCODE database (8). Furthermore, recent advances in whole-genome sequencing have led to the identification of new IncRNAs that are linked to cancer occurrence and progression (9-11).

LncRNA LOC285194, known as LSAMP antisense RNA3 and tumor suppressor candidate 7 , is a newly determined lncRNA located on chromosome 3q13.31. It consists of four exons of about $2.1 \mathrm{~kb}$ in length and was first discovered to inhibit tumor progression in osteosarcoma (12). Similar to most lncRNAs that impact cellular functions through interactions with chromatin remodeling proteins and regulation of other non-coding RNAs, the biological function of LOC298194 has been shown to mediate suppression of cell growth in part through suppression of microRNAs (13). Recent studies have shown that down-regulation of LOC285194 is associated with several cancers (such as colorectal, esophageal, gastric, pancreatic, and hepatocellular carcinoma) and their prognosis $(11,14-17)$. The tumor 
suppression function of LOC285194 has been demonstrated by knockdown experiments, showing that its knockdown can lead to increased cell proliferation (12).

The regulatory mechanism and function of LOC285194 in human cancer is widely unknown, which warrants further investigation. Meanwhile, technological advances in DNA sequencing have suggested that when mutated, cancer drivers (whether it is an oncogene or tumor suppressor) show a broad spectrum of tissue specificity. However, there is no existing evidence of the aberrant expression of LOC285194 and its underlying molecular mechanisms involved in ovarian carcinogenesis and progression. Thus, the aim of this study was to explore the function and clinical significance of LOC285194 in EOC and its possible underlying mechanism of tumorigenesis.

\section{Materials and Methods}

Cell lines and cell culture. Human epithelial ovarian cancer (EOC) cell lines A2780 was purchased from the European Collection of Cell Cultures (ECACC, Sigma-Aldrich, St. Louis, MO, USA) and cultured in RPMI-1640 medium (Gibco, Thermo Fisher Scientific Inc., Waltham, MA, USA) supplemented with $10 \%$ fetal bovine serum (FBS, Gibco) and 1\% penicillin/streptomycin (pen/strep, Gibco). SKOV3 cell line was obtained from the Korean Cell Line Bank (KCLB, Seoul, Republic of Korea). OVCA433, OVCA429, and TOV112D cell lines were provided by the Korea Gynecologic Cancer Bank through the Bio \& Medical Technology Development Program of the Minister of Science, Information and Communication Technology and Future Planning (MSIP), Korea and cultured in Dulbecco's modified Eagle's medium. These cell lines were maintained at $37^{\circ} \mathrm{C}$ with $5 \% \mathrm{CO}_{2}$. The culture medium was replaced with fresh medium every 2-3 days. The passage number of cells was $<20$ in all experiments.

Quantitative real-time polymerase chain reaction ( $q R T-P C R)$. Total RNA was extracted from cancerous/non-cancerous specimens and cell lines with TRIzol ${ }^{\circledR}$ reagent (Invitrogen, Thermo Fisher Scientific Inc., Waltham, MA, USA). Total RNAs $(1 \mu \mathrm{g})$ were reverse transcribed into first-strand cDNAs by using a reverse transcription reagent kit (Invitrogen) according to the manufacturer's protocol. qRT-PCR was carried out in 96-well blocks with a StepOnePlus ${ }^{\mathrm{TM}}$ Real-Time PCR System (Applied Biosystems, Thermo Fisher Scientific Inc., Waltham, MA, USA) using Power SYBR ${ }^{\circledR}$ Green Master Mix (Applied Biosystems) in a reaction volume of $20 \mu \mathrm{l}$. The following PCR program was used: an initial denaturation at $95^{\circ} \mathrm{C}$ for 2 min followed by 40 cycles of $15 \mathrm{~s}$ at $95^{\circ} \mathrm{C}$ and $60^{\circ} \mathrm{C}$ for $60 \mathrm{~s} .18 \mathrm{~S}$ rRNA gene was used as an internal control. PCR primer sequences were as follows: LOC285194: 5'-GGACCTGCCCTCCATTCTAT-3' (sense) and 5'GCATGGCATTTTCTCT-CCTC-3' (antisense) 18S rRNA: 5'GTAACCCGTTGAACCCCATT-3' (sense) and 5'-CCATCCA ATCGGTAGTAGCG-3' (antisense). Changes in expression levels were determined using the comparative cycle threshold $(2-\Delta \Delta \mathrm{Ct})$ method.

Small interfering RNA (siRNA) transfection. LOC285194 small interfering RNA (siRNA) duplex was purchased from Genolution (Genolution Pharmaceuticals Inc., Seoul, Republic of Korea). Target sequences for LOC285194 siRNAs were as follows: LOC285194
siRNA, 5'-GAGGAAAGAAGCAUACAUCUU-3'. Transfections were performed with either 6-well, 12-well, or 96-well culture plates (Nunc, Denmark). Ovarian cancer cells were harvested and $1 \times 10^{6}$ (6-well), $5 \times 10^{2}$ (12-well) or $5 \times 10^{3}$ (96-well) cells were seeded to each well. These short RNA duplexes were transfected into cells using lipofectamine RNAiMAX transfection reagent (Invitrogen) in Opti-MEM I Reduced Serum medium (Gibco). The lipofectamine reagent and LOC285194 siRNA were diluted in OptiMEM at room temperature. Diluted RNAi duplex was then diluted with lipofectamine RNAiMAX. This siRNA-lipid complex was incubated at room temperature for $10 \mathrm{~min}$ and added into plates. After 4-6 h, culture medium was changed and cells were incubated at $37^{\circ} \mathrm{C}$ for 2 days.

Cell proliferation assay. Cell proliferation was evaluated using a Cell Counting Kit-8 (CCK-8) assay (Dojindo Molecular Technologies Inc., Kumamoto, Japan) according to the manufacturer's instructions. LOC285194 siRNA-transfected cells were seeded into 96-well flatbottomed plates at a density $5 \times 10^{3}$ cells per well in $200 \mu$ of culture medium. These cells were incubated overnight to allow for cell attachment and recovery, after which they were transfected with siLOC285194 for 24, 48, 72, and $96 \mathrm{~h}$. Then, $20 \mu \mathrm{l}$ of CCK-8 solution was added to each well of the plate. After incubation at $37^{\circ} \mathrm{C}$ for $1 \mathrm{~h}$, absorbance was measured at $450 \mathrm{~nm}$ using a microplate reader.

Wound healing assay. Cells were seeded into 6-well culture plates with serum-enriched medium and allowed to grow to $90 \%$ confluency. The serum-containing medium was removed and then serum-starved overnight. When cell confluence reached $100 \%$, artificial wounds were created by scratching the monolayer with a $200 \mu$ pipette tip. After scratching, cells were washed twice with $1 \times$ PBS to remove floating cells and fresh serum-free medium was added. Wound healing was investigated at 0,24 , and $48 \mathrm{~h}$ and captured with a microscope (Olympus, Japan).

ADP/ATP ratio and ROS production assay. Intracellular ADP and ATP contents were measured using an ADP/ATP ratio assay kit (ab65313, Abcam, Waltham, MA, USA), according to the manufacturer's instructions. A2780 and SKOV3 cells transfected with siLOC285194 were washed with DPBS and plated into a 96well plate at a density of $1 \times 10^{4}$ cells/well and incubated for $24 \mathrm{~h}$. The relative light unit (RLU) value was recorded by LS50B luminescence spectrometer (PerkinElmer, Waltham, MA, USA). The reactive oxygen species (ROS) levels in A2780 and SKOV3 cells were evaluated using ROS detection kit (ENZ-51011, ENZO, Broomfield, CO, USA). The day before the experiment, cells were seeded in 96-well black plates at a density of $1 \times 10^{4}$ cells per well. The $\mathrm{H}_{2}$ DCFDA dye was added at a concentration of $10 \mu \mathrm{M}$ and the reaction mixture was incubated at $37^{\circ} \mathrm{C}$ for $1 \mathrm{~h}$ in the dark. Fluorescence was measured using a fluorescence microplate reader at excitation and emission wavelengths ranging from $488 \mathrm{~nm}$ to $520 \mathrm{~nm}$.

Caspase-3/7 activity assay. Caspase-3/7 activity was assessed using a Caspase-Glo ${ }^{\circledR} \quad 3 / 7$ assay (Promega, Madison, WI, USA). LOC285194, A2780, and SKOV3 cells were seeded into 96-well white plates at a density of 5,000 cells/well and incubated at $37^{\circ} \mathrm{C}$ overnight prior to treatment. The plates were removed from the incubator and allowed to equilibrate at room temperature for 30 min. Then, $100 \mu \mathrm{l}$ of Caspase-Glo reagent was added directly to 
A

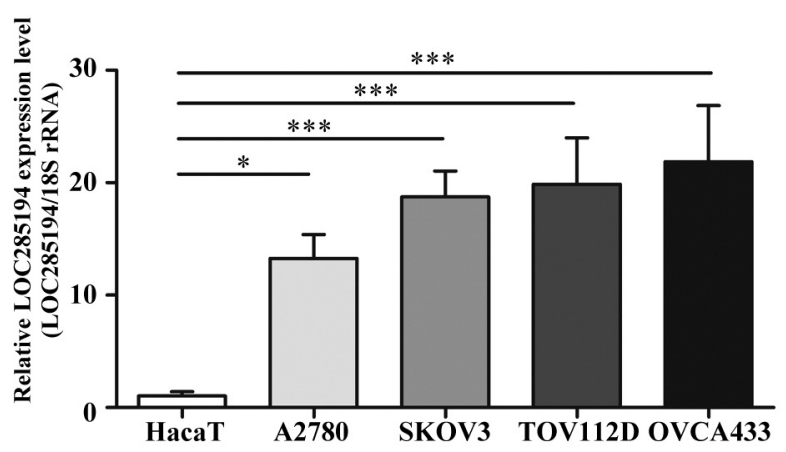

C

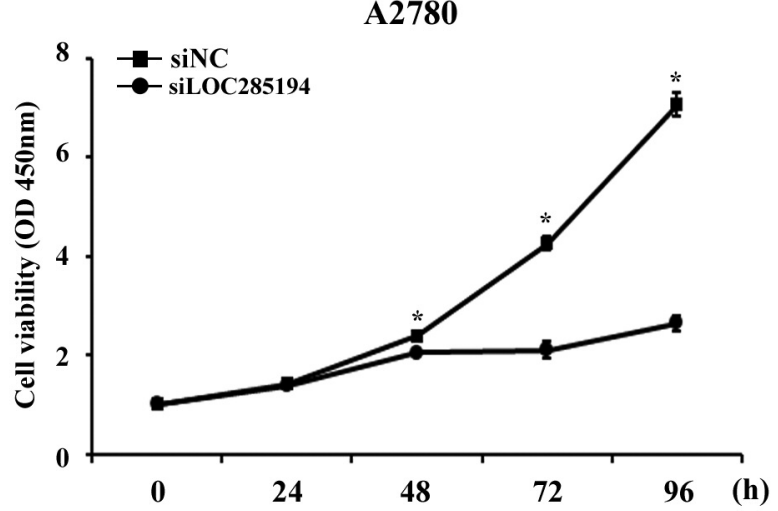

$\mathbf{E}$

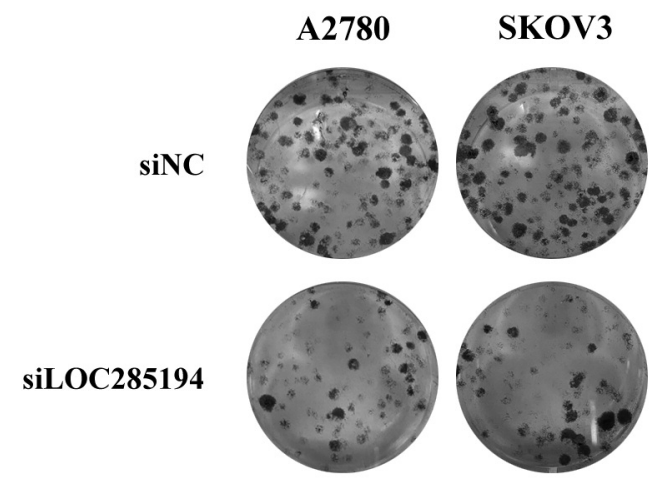

B

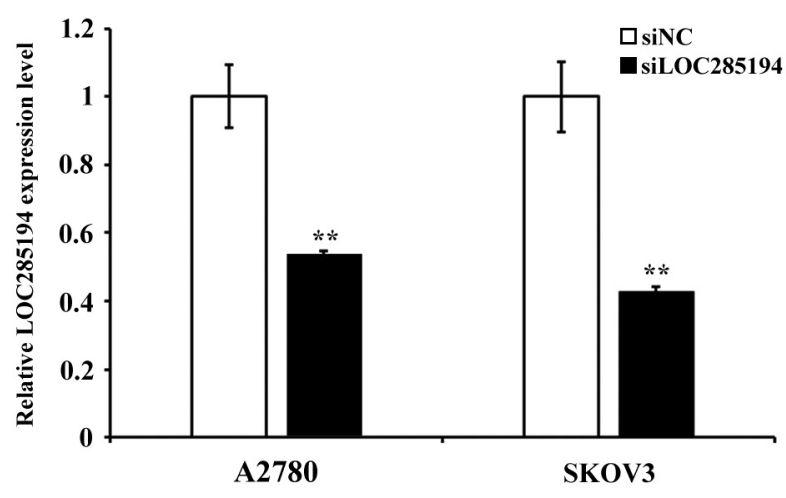

D

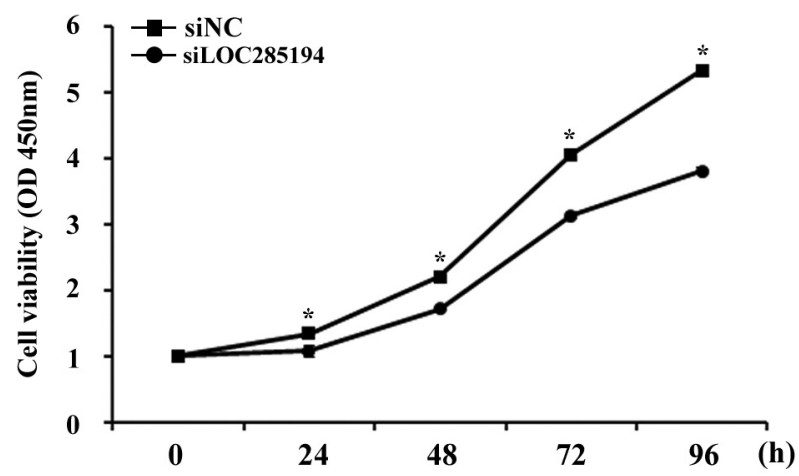

$\mathbf{F}$

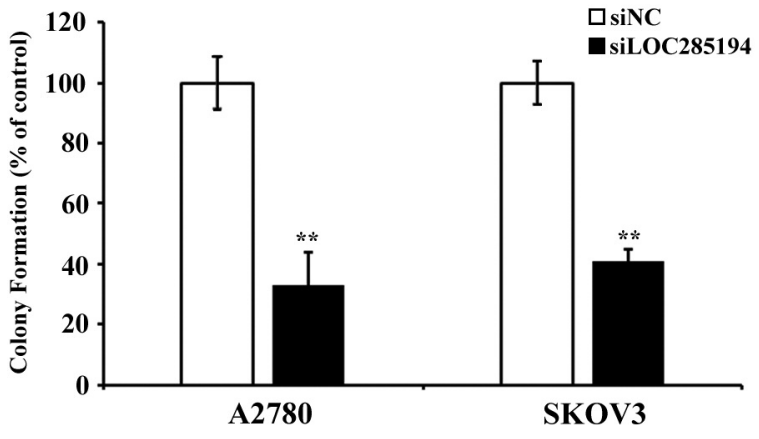

Figure 1. LOC285194 expression in ovarian cancer cell lines and knockdown of LOC285194 decreased cell proliferation. (A) The expression of LOC285194 in ovarian cancer cells (A2780, SKOV3, TOV112D, OVCA433) was significantly increased compared to control. (B) Quantitative realtime PCR analysis of LOC285194 levels confirmed that LOC285194 was silenced successfully by siRNA. (C, D) CCK-8 assays of A2780 and SKOV3 cells transfected with siLOC285194. (E) Clonogenic assay performed in A2780 and SKOV3 cell lines after siLOC285194 transfection. The representative colony formation images are shown for each cell line. (F) Colony formation of control and siLOC285194 transfected A2780 and SKOV3 cells. The percentage of colony formation was significantly decreased in siLOC285194 cells compared to control. Data represent the mean \pm standard error of mean from three independent experiments. ${ }^{*} p<0.05, * * p<0.01,{ }^{* * *} p<0.001$. si: Small interfering RNA; NC: negative control.

each well and incubated for $2 \mathrm{~h}$ at room temperature. The luminescence of each sample was recorded using a microplate luminometer (Berthold, Oak Ridge, TN, USA).
Western blot assay. Whole cell extracts containing total proteins were isolated using RIPA Lysis and Extraction buffer (Thermo Fisher Scientific, Inc.). Concentrations of proteins were quantified using a 
A

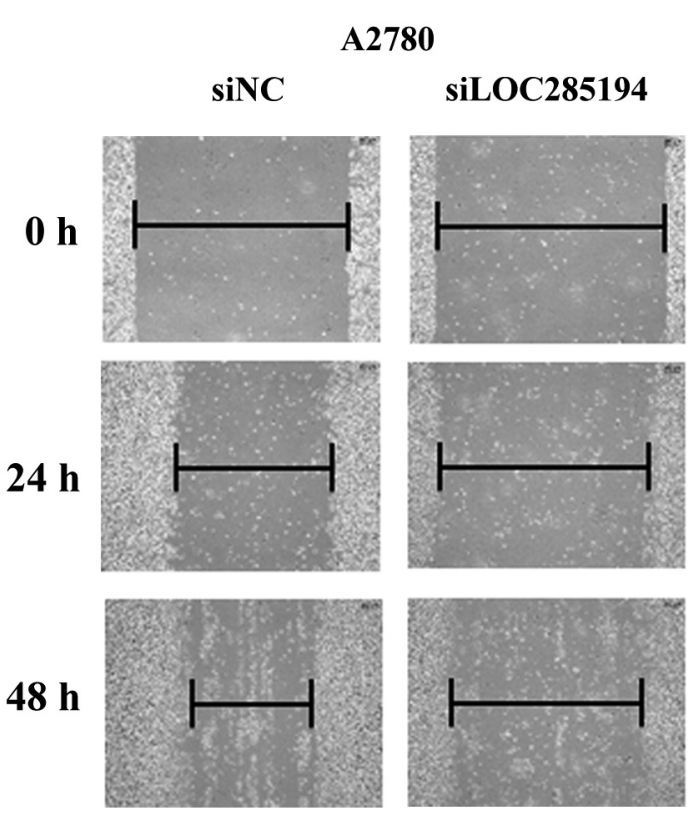

C

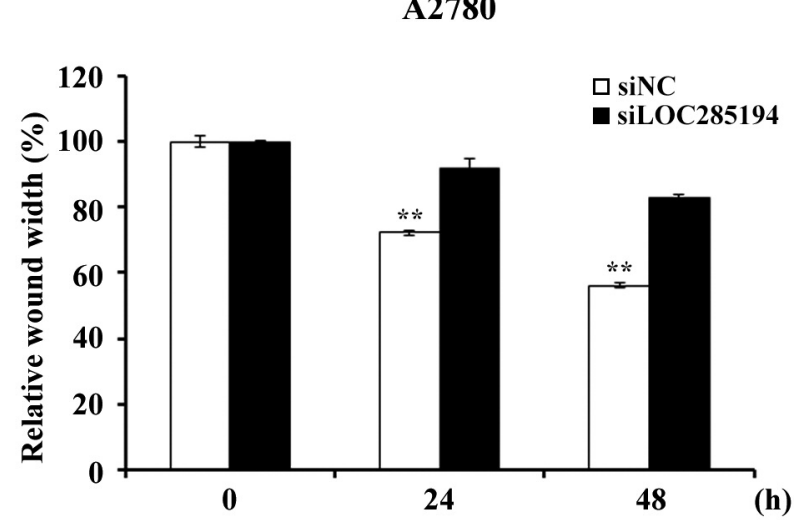

B

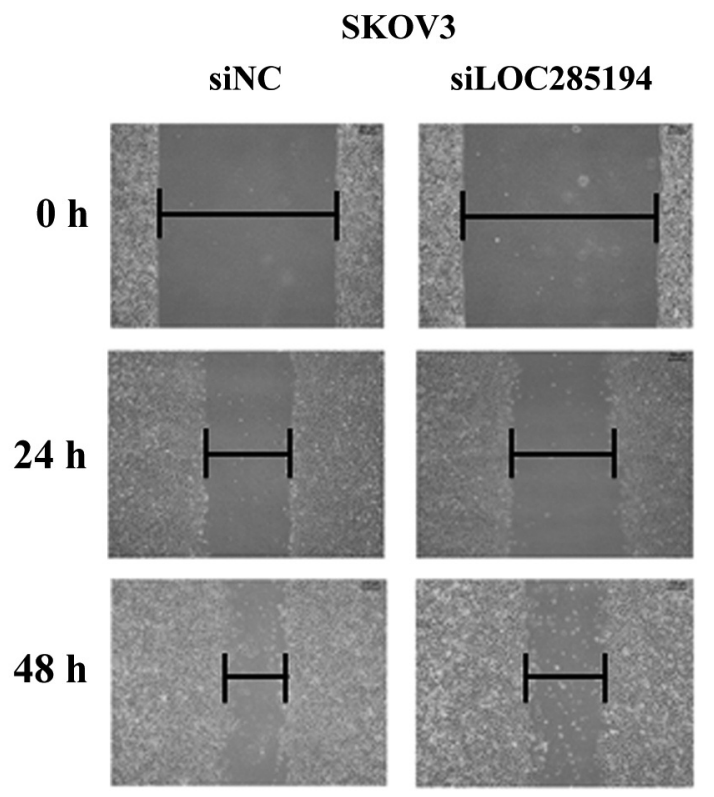

D

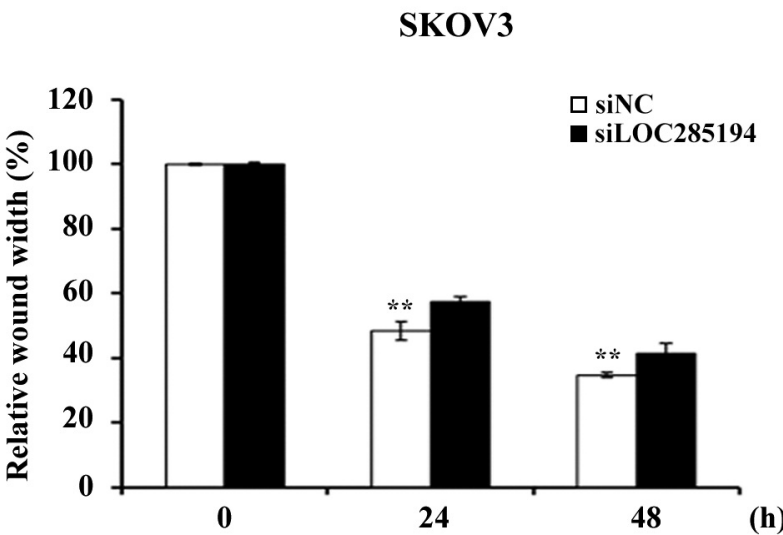

Figure 2. Silencing of LOC285194 inhibited cell migration ability in epithelial ovarian cancer cells. (A, B) The images of cells at healing sites at 24 and 48 h after scratching in A2780 and SKOV3 cells. In vitro cell migration was analyzed by wound healing assay to determine migration in siLOC285194-transfected A2780 and SKOV3 cells at 0,24 and $48 \mathrm{~h}$. Cell migration was visualized by a light microscope. (C, D) Wound area was determined by the rate of reduced scratched area. Wound width measurements at each time point in A2780 and SKOV3 cells were analysed by ImageJ software. The data are mean \pm SEM of three independent experiments performed in triplicate. ${ }^{*} p<0.01$. si: Small interfering RNA; NC: negative control.

Pierce BCA Protein Assay Kit (Thermo Fisher Scientific Inc.). Protein samples $(20 \mu \mathrm{g})$ were separated on $12 \%$ sodium dodecyl sulfate-polyacrylamide gel electrophoresis gels (SDS-PAGE) and then transferred electrophoretically onto polyvinylidene difluoride membranes (Millipore, Burlington, MA, USA). These membranes were blocked with $5 \%$ skimmed milk at room temperature for $1 \mathrm{~h}$ and incubated with a primary antibody against caspase-3, caspase-9, PARP, STAT-3, NF-kB, Fas, FasL, and $\beta$-actin at $4^{\circ} \mathrm{C}$ overnight. The membrane was washed three times with $1 \mathrm{X}$ Tris-Buffered Saline, $0.1 \%$ Tween ${ }^{\circledR} 20$ Detergent (TBS-T) buffer and then incubated with horseradish peroxidase-conjugated secondary antibody $(1: 5,000)$ at room temperature for $2 \mathrm{~h}$. Protein bands were visualized with an enhanced chemiluminescence solution, followed by detection with medical x-ray film (Fuji Film, Japan).

Patient and tissue sample. A total of 93 ovarian cancer tissues and patients' serum samples were obtained from patients who underwent surgery at the Department of Obstetrics and Gynecology, Severance Hospital between 2004 and 2014. All serum and tissue samples were collected after the patients' consent. The control group consisted of normal ovarian epithelial tissue samples obtained from patients who underwent a simple hysterectomy or oophorectomy for benign 
A

A2780

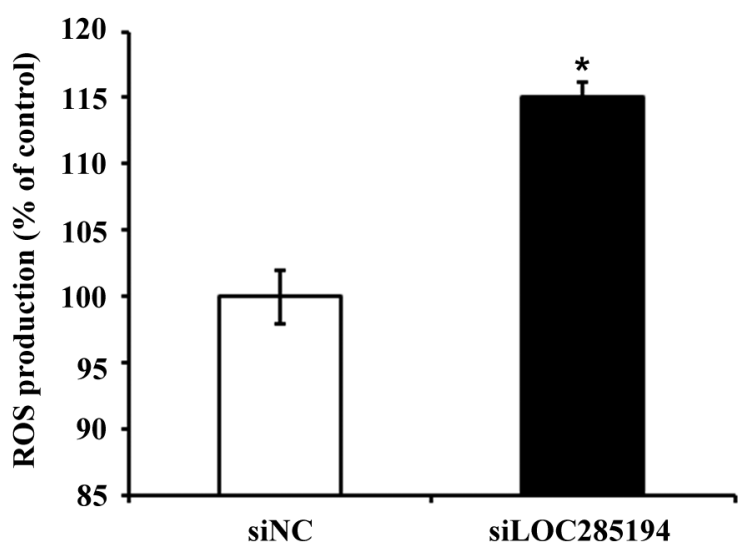

C

A2780

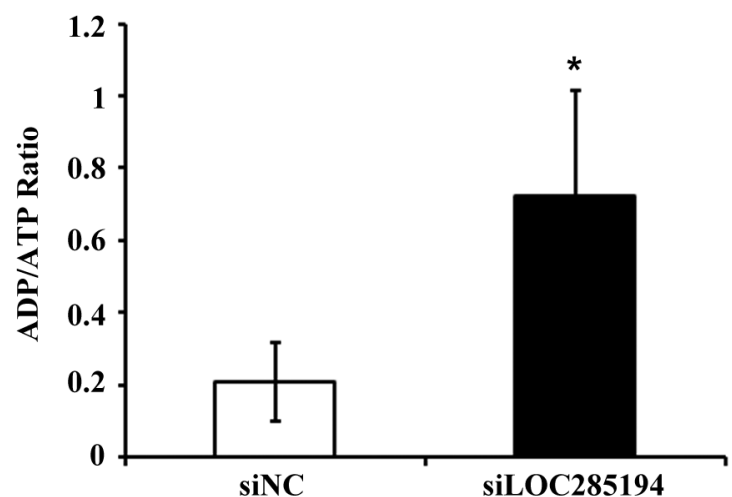

B

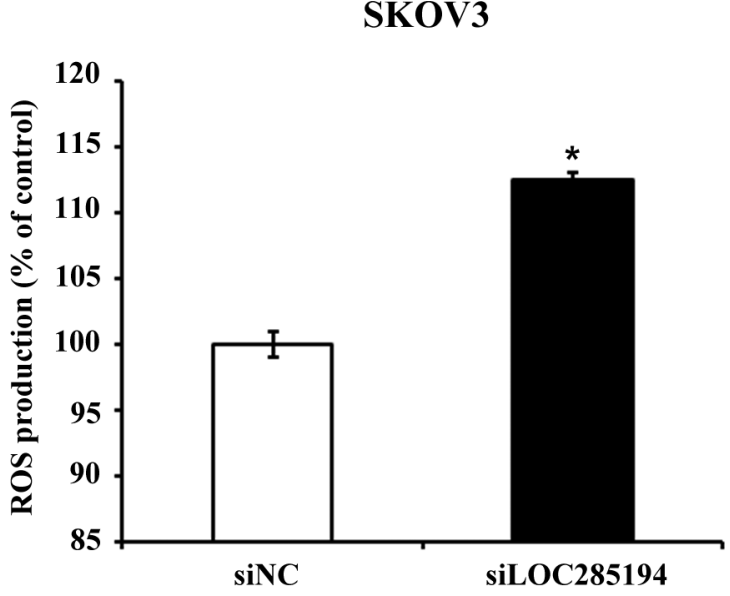

D

SKOV3

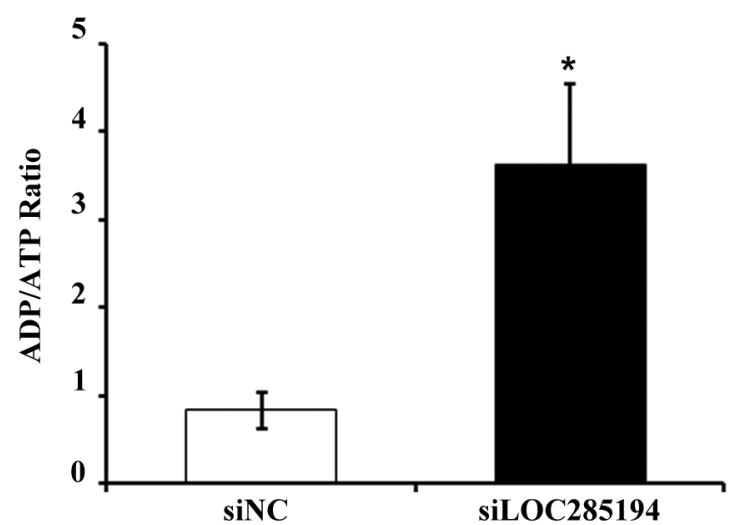

Figure 3. Increased reactive oxygen species (ROS) production and ADP/ATP ratio after silencing of LOC285194. (A, B) ROS production was determined using ROS detection assay kit. ROS production was increased in A2780 and SKOV3 cells following 48 h after transfection with siRNA $(p<0.05)$. (C, D) Effect of LOC285194 on the ADP/ATP ratio in epithelial ovarian cancer cells. ADP/ATP ratio was measured in control and siLOC285194-transfected cells. The ratio was markedly increased in LOC285194 knockdown cells. The results are expressed as mean \pm SEM from three independent experiments. ${ }^{*} p<0.05$. si: Small interfering $R N A ; N C$ : negative control.

uterine conditions. All tissue samples were immediately frozen in liquid nitrogen and stored at $-80^{\circ} \mathrm{C}$. Clinicopathologic and survival data were collected from the matching database. This study was approved by the Ethics Committee of Severance Hospital (IRB No. 4-2020-0612, IRB No. 4-2012-0363).

Statistical analysis. All statistical analyses were performed using SPSS Statistics 25 for Windows (SPSS Inc., Chicago, IL, USA). Student's $t$-tests and Fisher's exact tests were used to estimate associations between LOC285194 expression and clinicopathological features in patients with EOC. One-way ANOVA with post-hoc Tukey test was used for multiple comparisons among multiple samples. The median value (8.78) was set as a cutoff value between high and low expression. For in vitro analysis, all experiments were performed in triplicate and data representing the mean and standard error were analyzed by student's $t$-test. Progression-free survival (PFS) and overall survival (OS) were assessed with the Kaplan-
Meier method. Receiver operating characteristic (ROC) curves were used to compare the sensitivity and specificity of LOC285194. $p<0.05$ was considered as statistically significant.

\section{Results}

Knockdown of LOC285194 suppresses ovarian cancer cell proliferation in vitro. LOC285194 transcript levels in four ovarian cancer cell lines were analyzed by qRT-PCR. Higher LOC285194 expression levels were found in all ovarian cancer cells lines compared to control cells (Figure 1A). LOC285194-targeted siRNA was transfected into A2780 and SKOV3 cells. Compared with control cells, A2780 and SKOV3 cells transfected with siRNA showed remarkably decreased expression levels of LOC285194 (Figure 1B). 
A

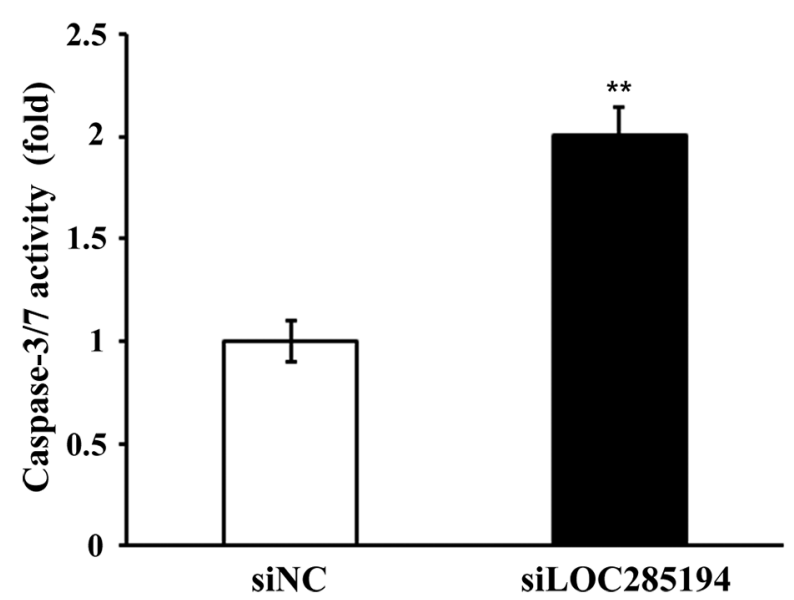

B

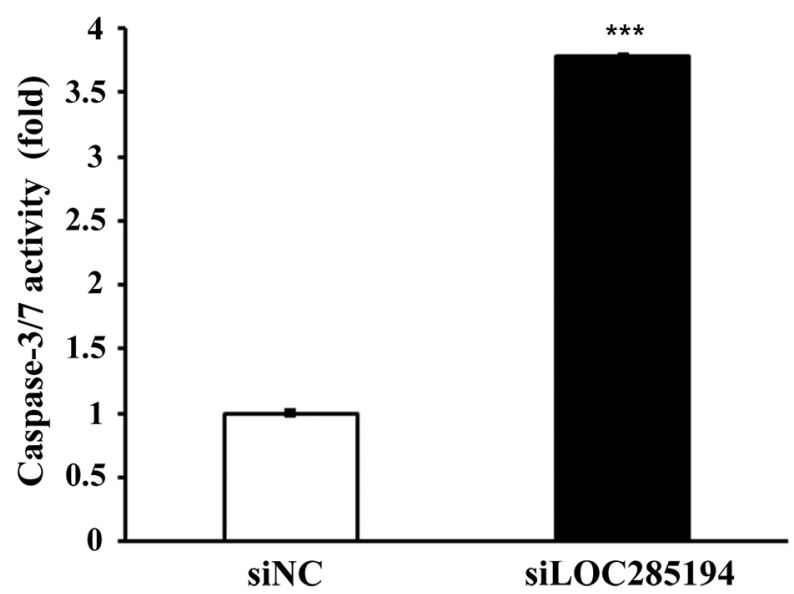

Figure 4. Effects of caspase-3/7 activity in A2780 and SKOV3 cells. The caspase 3/7 activity was measured using Caspase-Glo 3/7 assay. The caspase activities were expressed as fold increase relative to control cells. Data represent the mean $\pm S E M$ of three experiments. **p<0.01 and ***p<0.001. si: Small interfering RNA; NC: negative control.
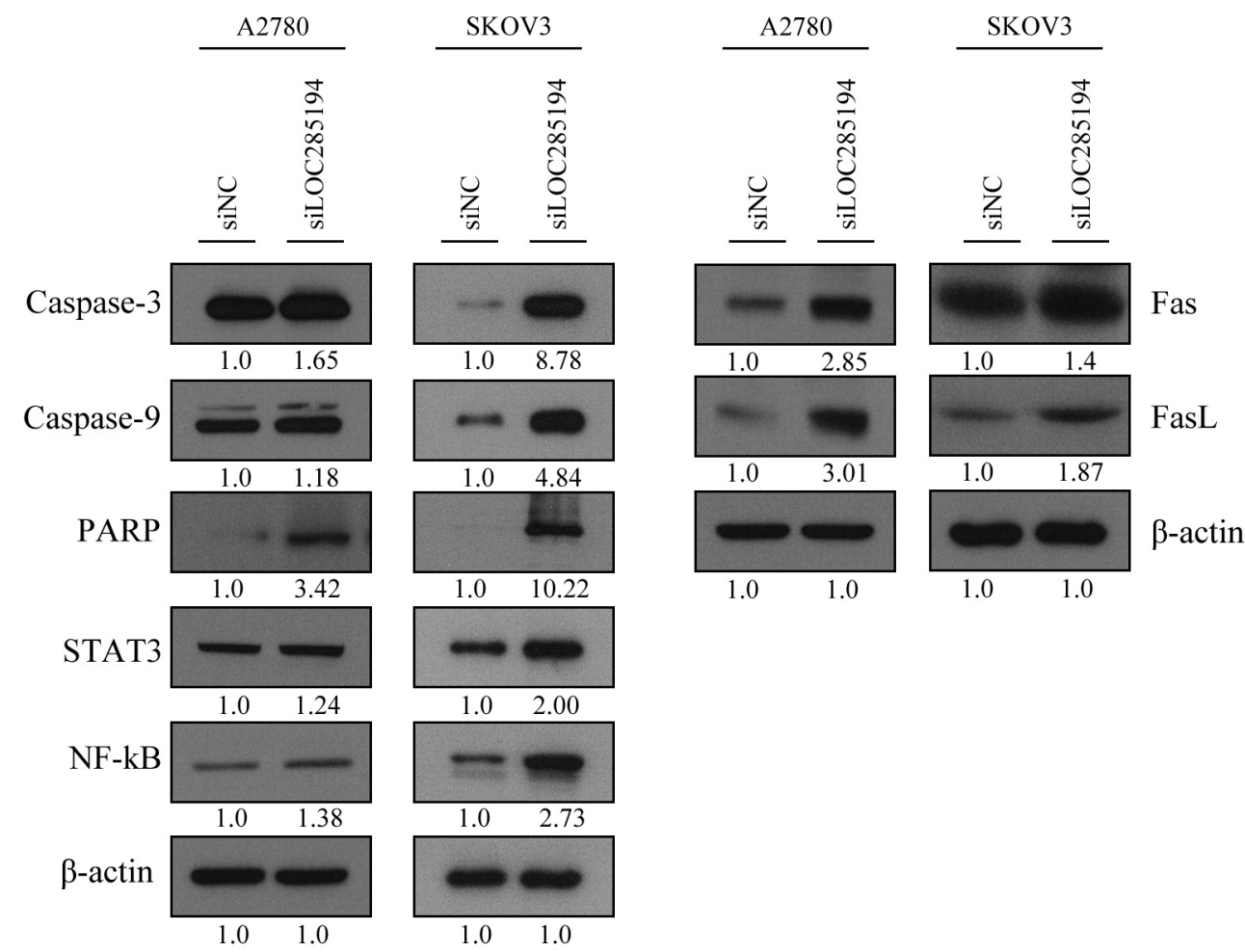

Figure 5. Western blot analysis of apoptosis-related proteins in A2780 and SKOV3 cells. A2780 and SKOV3 cells were transfected with control or siLOC285194 for 48 h. The expression of caspase-3, caspase-9, PARP, Fas, and FasL proteins increased after knockdown of LOC285194. Si: Small interfering RNA; NC: negative control.

Silencing of LOC285194 inhibited cell proliferation of A2780 and SKOV3. LOC285194 knockdown resulted in decreased cell proliferation (Figure 1C and D). According to colony formation assay, knockdown of LOC285194 significantly $(p<0.001)$ reduced the numbers of colony formation by more than $30 \%$ (Figure $1 \mathrm{E}$ and $\mathrm{F}$ ). Abilities of 
A
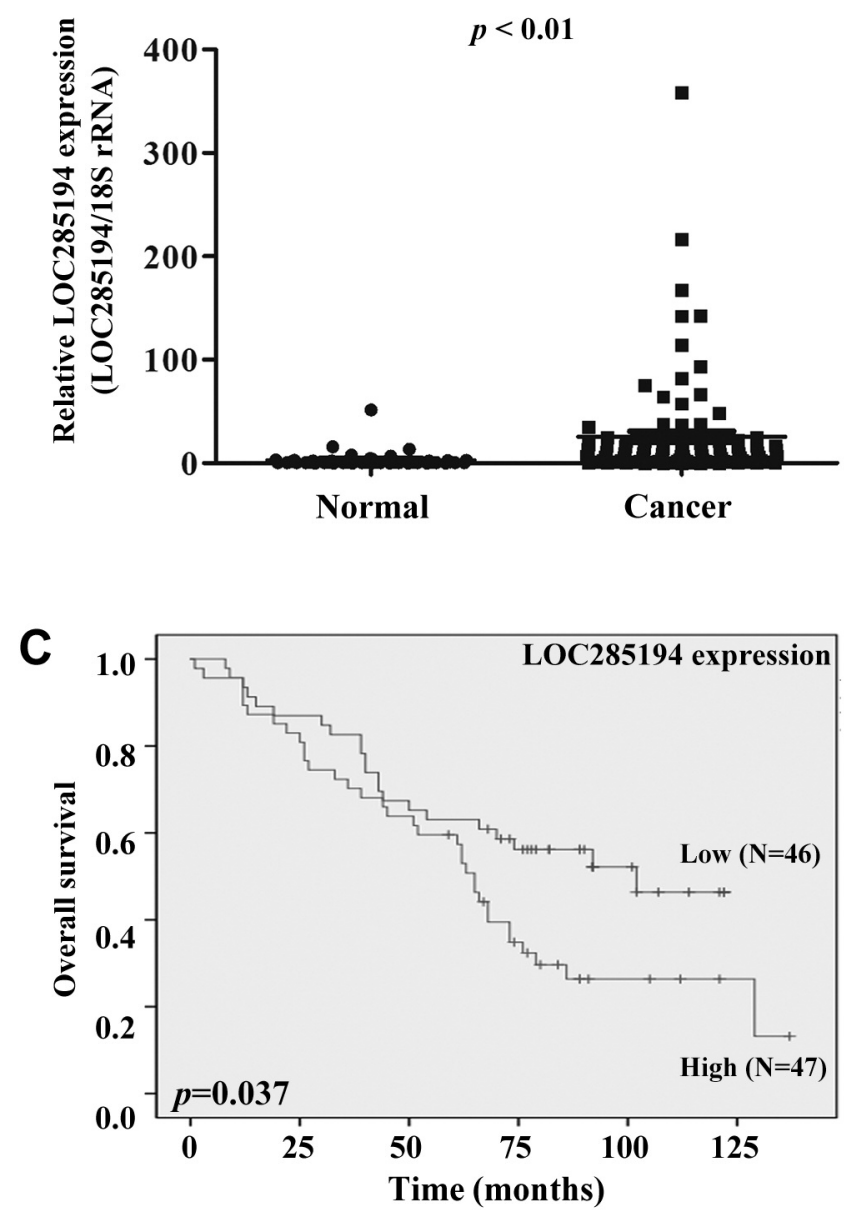

B
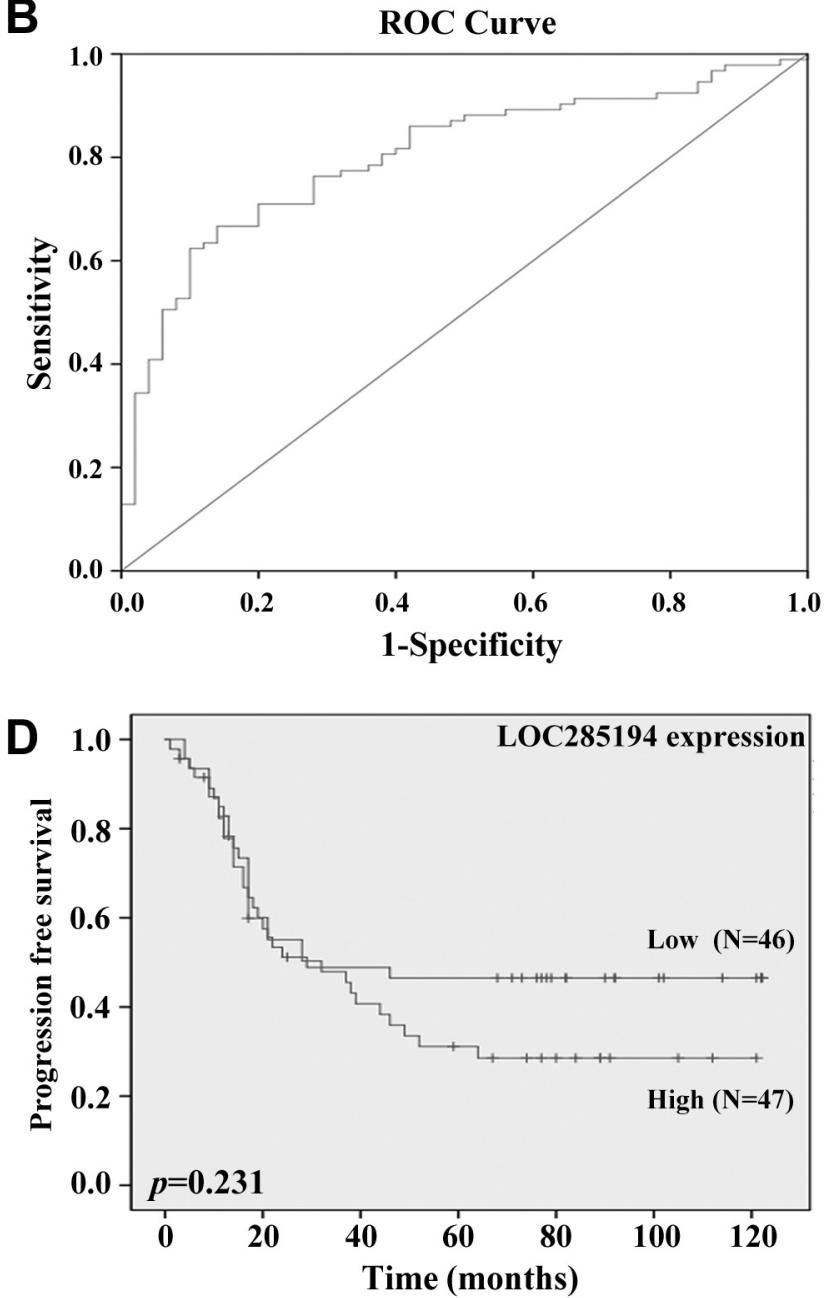

Figure 6. Expression status and prognostic value of LOC285194 in epithelial ovarian cancer. (A) The relative expression of LOC285194 in epithelial cancer tissues $(n=93)$ and non-cancerous tissues $(n=50)$. LOC285194 expression was significantly higher in ovarian cancer tissues compared to non-cancerous tissues. Relative expression of LOC285194 in ovarian cancer and matched normal tissues was determined by qRT-PCR. (B) ROC curve for prognostic predictions by LOC285194 expression. The area under the curve of LOC285194 alone was 0.803 (95\%CI=0.730-0.875, p=0.037). (C, D) Kaplan-Meier survival plot for the overall (OS) and progression-free survival (PFS) of patients according to different expression levels of LOC285194. High LOC285194 expression was associated with poor OS.

A2780 and SKOV3 cell lines to form colonies were increased when LOC285194 was suppressed. Taken together, these results showed that down-regulation of LOC285194 could lead to decreased cell proliferation and colony forming ability of ovarian cancer cells A2780 and SKOV3.

LOC285194 knockdown prevents cell migration. Larger widths of wounds in siLOC285194-transfected A2780 and SKOV3 cells were shown compared to control (Figure 2A and $\mathrm{B})$. Wound healing by SKOV3 control cells was faster such that the wound area was reduced $60 \%$ at 48 h. A2780 cells reduced the wound area by $45 \%$ at $48 \mathrm{~h}$ (Figure $2 \mathrm{C}$ and D). Silencing of LOC285194 impeded wound closure in
A2780 and SKOV3. Collectively, down-regulation of LOC285194 decreased ovarian cancer cell proliferation compared to control cells.

Increased cell death following LOC285194 knockdown is due to ROS production. To determine whether LOC285194 might have functions in oxidative stress-induced apoptosis of ovarian cancer, ROS levels were quantified in ovarian cancer and LOC285194 knockdown cells (A2780, SKOV3). ROS production was found to be increased upon silencing of LOC285194 compared to that in non-transfected ovarian cancer cells based on $\mathrm{H}_{2}$ DCF-DA fluorescent assay (Figure $3 \mathrm{~A}$ and $\mathrm{B})$. In addition, the ADP/ATP ratio was significantly 
increased in LOC285194 knockdown cells (Figure 3C and D). Thus, silencing of LOC285194 enhanced ROS production and ADP/ATP ratios in A2780 and SKOV3 cells, leading to increased cell apoptosis.

LOC285194 modulates apoptosis-related proteins and caspase activity in ovarian cancer cells. The effects of caspase-3/7 activity in A2780 and SKOV3 cells are shown as fold increase relative to control cells (Figure 4). To determine whether the observed changes in expression were related to apoptosis, components of apoptosis signaling in ovarian cancer cells were evaluated by western blot. Expression levels of caspase-3, caspase-9, STAT3, NF-kB, and PARP protein as well as Fas/FasL were all induced after knockdown of LOC285194 (Figure 5). These results suggest that lncRNA LOC285194 regulates the levels of apoptosisassociated proteins in SKOV3 cells.

LOC285194 is over-expressed in EOC tissues and its overexpression is correlated with poor prognosis. By quantitative real-time polymerase chain reaction (qRT-PCR) analysis, lncRNA LOC285194 expression levels were found to be significantly $(p<0.01)$ higher in cancer tissues than in control (non-cancerous) tissues (Figure 6A). The area under the receiver operating characteristic (ROC) curve was 0.803 $(p<0.01)$ (Figure 6B). ROC curve analysis showed that the expression of LOC285194 was a predictor of prognosis of EOC patients. Clinicopathological data were compared between low and high LOC285194 expression groups (Table I). There was no significant difference in age, stage, histological subtype, lymph node metastasis, or CA125 level between high and low LOC285194 expression groups. KaplanMeier survival analysis indicated that patients with high LOC285194 levels had a poorer overall survival compared to those with low LOC285194 expression (65 months vs. 102 months, $p<0.05$; Figure 6C) and not PFS (Figure 6D). In multivariate Cox regression analysis for survival, LN metastasis and LOC285194 expression were significant predictors of overall survival (OS). High LOC285194 expression in tumor tissues was an independent predictor of OS $\quad(\mathrm{HR}=5.880,95 \% \mathrm{CI}=1.894-18.252, \quad p=0.002)$, after controlling for age, stage, grade, cell type, and lymph node metastasis (Table II).

\section{Discussion}

In this study, the knockdown of LOC285194 expression demonstrated decreased cell proliferation, migration, invasion in ovarian cancer cells and increased cell apoptosis. Western blot analysis showed that LOC285194 knockdown regulates apoptosis by altering the expression of apoptosisrelated proteins. Likewise, LOC285194 expression levels were found to be higher in EOC tissues than in noncancerous
Table I. LOC285194 expression and clinicopathological characteristics of patients.

\begin{tabular}{|c|c|c|c|c|}
\hline & \multicolumn{3}{|c|}{ LOC285194 } & \multirow[b]{2}{*}{$p$-Value } \\
\hline & Low $(n=46)$ & High $(n=47)$ & Total & \\
\hline Age & & & & 0.701 \\
\hline$<60$ & $32(69.6 \%)$ & $32(68.1 \%)$ & 65 & \\
\hline$\geq 60$ & $13(28.3 \%)$ & $15(31.9 \%)$ & 28 & \\
\hline Stage & & & & 0.693 \\
\hline I & $12(26.1 \%)$ & $11(23.4 \%)$ & 23 & \\
\hline II & $4(8.7 \%)$ & $8(17.0 \%)$ & 12 & \\
\hline III & $24(52.2 \%)$ & $22(46.8 \%)$ & 46 & \\
\hline IV & $6(13.0 \%)$ & $6(12.8 \%)$ & 12 & \\
\hline Grade & & & & 0.078 \\
\hline 1 & $5(10.9 \%)$ & $3(6.4 \%)$ & 8 & \\
\hline 2 & $13(28.3 \%)$ & $24(51.1 \%)$ & 37 & \\
\hline 3 & $28(60.9 \%)$ & $20(42.6 \%)$ & 48 & \\
\hline Histological type & & & & 0.648 \\
\hline Serous & $30(65.2 \%)$ & $33(70.2 \%)$ & 63 & \\
\hline Mucinous & $3(6.5 \%)$ & $2(4.3 \%)$ & 5 & \\
\hline Endometrioid & $4(8.7 \%)$ & $7(14.9 \%)$ & 11 & \\
\hline Clear cell & $8(17.4 \%)$ & $4(8.5 \%)$ & 12 & \\
\hline Others & $1(2.2 \%)$ & $1(2.2 \%)$ & 2 & \\
\hline LN metastasis & & & & 0.148 \\
\hline Absent & $20(43.5 \%)$ & $28(59.6 \%)$ & 48 & \\
\hline Present & $26(54.5 \%)$ & $19(40.4 \%)$ & 45 & \\
\hline CA125 & & & & 0.384 \\
\hline$<35$ & $4(8.7 \%)$ & $2(4.3 \%)$ & 6 & \\
\hline$\geq 35$ & $42(91.3 \%)$ & $45(95.7 \%)$ & 87 & \\
\hline Recurrence & & & & 0.174 \\
\hline No & $28(60.9 \%)$ & $22(46.8 \%)$ & 50 & \\
\hline Yes & $18(39.1 \%)$ & $25(53.2 \%)$ & 43 & \\
\hline
\end{tabular}

LN: Lymph node.

ovarian tissues. Furthermore, increased LOC285194 expression was associated with lymph node metastasis and poor overall patient survival. Clinical data revealed adverse prognosis for groups with high LOC285194 expression. These findings suggest that LOC285194 may promote tumor progression by regulating apoptosis protein markers.

Previous studies on other human carcinomas revealed that LOC285194 is a tumor suppressor rather than a promoter. One of the suggested mechanism is that LOC285194 binds to p53 protein, which activates apoptosis and regulates the expression of apoptosis, DNA repair, cellular senescence and growth arrest related genes (13). Several recent studies described lncRNAs as negative regulators of apoptosis in different types of tumors. For example, a lncRNA called AFAP1-AS1, derived from the antisense strand of the AFAP1 coding gene, showed hypomethylation and upregulation in esophageal cancer tissues and cell lines (5). Silencing of AFAP1-AS1 by siRNA was shown to induce apoptosis in the relevant cell line, suggesting that AFAP1-AS1 can modulate both proliferation and programmed cell death of esophageal 
Table II. Univariate and multivariate Cox regression analyses for factors associated with progression free and overall survival.

\begin{tabular}{|c|c|c|c|c|c|c|c|c|c|c|c|c|}
\hline \multirow{3}{*}{ Characteristics } & \multicolumn{6}{|c|}{ Progression-free survival } & \multicolumn{6}{|c|}{ Overall survival } \\
\hline & \multicolumn{3}{|c|}{ Univariate analysis } & \multicolumn{3}{|c|}{ Multivariate analysis } & \multicolumn{3}{|c|}{ Univariate analysis } & \multicolumn{3}{|c|}{ Multivariate analysis } \\
\hline & HR & $95 \% \mathrm{CI}$ & $p$-Value & HR & $95 \% \mathrm{CI}$ & $p$-Value & HR & $95 \% \mathrm{CI}$ & $p$-Value & HR & $95 \% \mathrm{CI}$ & $p$-Value \\
\hline \multicolumn{13}{|l|}{ Age } \\
\hline$\leq 56 v s .>56$ & 0.629 & $0.263-1.506$ & 0.298 & & & & 1.313 & $0.538-3.204$ & 0.549 & & & \\
\hline \multicolumn{13}{|l|}{ Stage } \\
\hline $3-4$ vs. $1-2$ & 5.494 & $2.206-13.684$ & $<0.001$ & 1.702 & $0.333-8.692$ & 0.523 & 4.851 & $1.977-11.969$ & 0.001 & 3.688 & $0.852-15.969$ & 0.081 \\
\hline \multicolumn{13}{|l|}{ Grade } \\
\hline 3 vs. 1-2 & 0.781 & $0.340-1.790$ & 0.558 & & & & 0.850 & $0.370-1.953$ & 0.702 & & & \\
\hline \multicolumn{13}{|l|}{ Cell type } \\
\hline $\begin{array}{l}\text { Non-serous } \\
v s . \text { serous }\end{array}$ & 0.310 & $0.126-0.765$ & 0.091 & & & & 0.356 & $0.145-0.871$ & 0.084 & & & \\
\hline \multicolumn{13}{|l|}{$\mathrm{LN}$ metastasis } \\
\hline $\begin{array}{l}\text { Positive } v s \text {. } \\
\text { negative }\end{array}$ & 13.000 & $4.557-37.085$ & $<0.001$ & 16.125 & $3.484-74.625$ & $<0.001$ & 4.500 & $1.820-11.124$ & 0.001 & 4.615 & $1.203-17.703$ & 0.026 \\
\hline \multicolumn{13}{|l|}{ Initial CA125 } \\
\hline$>35$ vs. $\leq 35$ & 4.571 & $1.883-11.098$ & 0.101 & & & & 1.900 & $0.816-4.424$ & 0.137 & & & \\
\hline \multicolumn{13}{|l|}{$\begin{array}{l}\text { LOC285194 } \\
\text { expression }\end{array}$} \\
\hline High vs. low & 1.776 & $0.770-4.097$ & 0.178 & & & & 2.853 & $1.205-6.756$ & 0.017 & 5.880 & $1.894-18.252$ & 0.002 \\
\hline
\end{tabular}

LN: Lymph node; CI: confidence interval; HR: hazard ratio.

cancer cells. On the other hand, several reports demonstrated the pro-apoptotic effects of lncRNAs in different cancer cells such as growth arrest-specific 5 (GAS5) in prostate cancer and uc002mbe. 2 in hepatocellular carcinoma $(7,18)$ Likewise, lncRNAs are involved in the modulation of apoptosis both by promoting and inhibiting apoptotic pathways in cancer cells. In this context, the relationship between the expression of LOC285194 and clinical prognostic data shown in this study were partly different from previous studies on LOC285194. On Cox regression model, stage, LN metastasis, and LOC285194 expression were associated with both PFS and OS on univariate analysis. Other variables such as grade, cell type, and initial CA125 did not show significant relationship with survival. These results may not be sufficiently convincing due to the relatively small sample size; however, similar findings were shown in a recent systematic review and meta-analysis in that age, stage, and grade were not associated with LOC285194 expression in cancers (19). Since there are no studies on LOC285194 in ovarian cancer, we feel the need for further study and validation in the future. Since EOC shows the poorest prognosis among gynecologic cancers, the role of lncRNA in the known factors of recurrence such as angiogenesis and lymphatic metastasis should be further investigated (20). Importantly, lncRNAs are known to have both pro-apoptotic and anti-apoptotic properties, and therefore unlike other studies in different cancers, LOC285194 acted as an oncogene rather than a tumor suppressor gene in our study.
This study demonstrated that LOC285194 promotes cell proliferation and migration via regulation of apoptosis-related proteins. However, the biological role of LOC285194 in cell proliferation and apoptosis of EOC is still unknown. Therefore, to investigate the molecular mechanism of LOC285194 in cell apoptosis, the level of p53 proteins was demonstrated using western blot assay after siRNA-mediated knockdown of LOC285194 in three ovarian cancer cell lines including A2780, SKOV3, and TOV112D (data not shown). p53 protein level was induced in siLOC285194 transfected A2780 and TOV112D cell except p53 null SKOV3 cells. Caspase-3/7 activity in A2780 and SKOV3 cells was increased relative to control cells, which indicated the activation of apoptosis process in the relevant cell lines. Subsequently, the expression levels of caspase-3, caspase-9, STAT3, NF-kB, and PARP protein as well as Fas/FasL were all shown to be induced after knockdown of LOC285194. These results suggest that lncRNA LOC285194 can control caspase-associated apoptosis proteins in ovarian cancer cells. In addition, we showed that LOC285194 regulates oxidative stress-induced apoptosis markers in ovarian cancer and relevant p53 modulation could be a possible underlying mechanism for cell death in EOC.

Based on the in vitro study results above using ovarian cancer cell lines, we aimed to demonstrate the role of LOC285194 in the clinical outcome and prognosis of EOC. Contrary to previous literature on LOC285194 in nongynecological cancers, the expression level of LOC285194 
was significantly higher in ovarian cancer tissues compared to that in non-cancerous tissues. Moreover, high LOC285194 expression was a significant factor for both PFS and OS. The different effect of LOC285194 on ovarian cancer progression in this study could be due to tissue differences. With recent advances in targeted DNA sequencing, there exists abundance in genetic information revealing the patterns and frequencies of mutations and copy number variations on the entire spectrum of cancers. Based on the results of the genomic analysis of primary cancers, it is hypothesized that tissue specificity is driven by the preexisting epigenetic environment across tissues (21). This means that oncogenes and tumor suppressor genes could only exert their effects when the epigenetic state allows a tissue to respond to the specific oncogenic signal. Different cells from different cancer types have distinct chromatin and proteomic states. Therefore, they may respond differently to a particular stimulation. For example, many prominent cancer genes such as von Hippel Lindau tumor suppressor $(V H L)$ in renal cancer, adenomatous polyposis coli $(A P C)$ in colorectal cancer, and KRAS in pancreas, colon, and lung cancer show broad patterns of gene and protein expression, but restricted cancer-associated mutations. These observations might be explained by the fact that cells from different developmental lineages differ greatly in their abilities to respond to growthpromoting events (22). Therefore, functional alterations in specific genes can promote tumorigenesis in some tissues whereas they are ineffective in other tissues. Moreover, IncRNAs are known to be more tissue specific than proteincoding genes, although the mechanisms that govern lncRNA transcription to determine higher tissue specificity remain unclear (23). A recent study using high-throughput functional analysis of lncRNA core promoters suggested that core promoter sequences play important roles in determining transcript tissue specificity (24). Tissue specificity in cancer remains to be extensively explored.

The limitation of this study is its retrospective nature. In addition, there is limited generalizability due to the fact that the results are from a single ethnic group. Whether there are variations among cancers due to tissue specificity or ethnic differences warrants further investigation. However, a large number of ovarian cancer patient cases and tissues were examined along with long-term clinical data such as survival. Studies addressing the role of LOC285194 in gynecological cancer are scarce and no studies examined the apoptosis-related modulation of lncRNA LOC285194 in EOC. To the best of our knowledge, this study is the first to explore the function and clinical significance of LOC285194 in EOC and its possible underlying mechanism of tumorigenesis. Additional studies are needed to confirm the molecular mechanism of tumorigenesis of LOC285194 for its use as a potential diagnostic and prognostic biomarker in EOC.
In conclusion, findings of the present study indicate that LOC285194 may have a role in promoting cancer progression by regulating apoptosis. Further studies are needed to elucidate the role of LOC285194 in ovarian carcinogenesis.

\section{Conflicts of Interest}

The Authors declare no conflicts of interest in relation to this study.

\section{Authors' Contributions}

Study design: GWY, JK, YTK; collection of data: GWY, JK, YTK; data analysis: GWY, JK, DWL, YTK; article preparation, editing, and review: GWY, JK, DWL, YTK.

\section{Acknowledgements}

This work was supported by the Basic Science Research Program of the National Research Foundation of Korea (NRF) funded by the Ministry of Education, Science, and Technology (grant number NRF-2018R1D1A1B07049578).

\section{References}

1 Cerk S, Schwarzenbacher D, Adiprasito JB, Stotz M, Hutterer GC, Gerger A, Ling H, Calin GA and Pichler M: Current status of long non-coding RNAs in human breast cancer. Int J Mol Sci 17(9): 1485, 2016. PMID: 27608009. DOI: 10.3390/ ijms 17091485

2 Pellecchia S, Sepe R, Decaussin-Petrucci M, Ivan C, Shimizu M, Coppola C, Testa D, Calin GA, Fusco A and Pallante P: The long non-coding RNA Prader Willi/Angelman Region RNA5 (PAR5) is downregulated in anaplastic thyroid carcinomas where it acts as a tumor suppressor by reducing EZH2 activity. Cancers (Basel) 12(1): 235, 2020. PMID: 31963578. DOI: 10.3390/ cancers 12010235

3 Harries LW: Long non-coding RNAs and human disease. Biochem Soc Trans 40(4): 902-906, 2012. PMID: 22817756. DOI: $10.1042 / B S T 20120020$

4 Zhao H, Zhang X, Frazão JB, Condino-Neto A and Newburger PE: HOX antisense lincRNA HOXA-AS2 is an apoptosis repressor in all trans retinoic acid treated NB4 promyelocytic leukemia cells. J Cell Biochem 114(10): 2375-2383, 2013. PMID: 23649634. DOI: 10.1002/jcb.24586

$5 \mathrm{Wu}$ W, Bhagat TD, Yang X, Song JH, Cheng Y, Agarwal R, Abraham JM, Ibrahim S, Bartenstein M, Hussain Z, Suzuki M, Yu Y, Chen W, Eng C, Greally J, Verma A and Meltzer SJ: Hypomethylation of noncoding DNA regions and overexpression of the long noncoding RNA, AFAP1-AS1, in Barrett's esophagus and esophageal adenocarcinoma. Gastroenterology 144(5): 956966.e4, 2013. PMID: 23333711. DOI: 10.1053/j.gastro.2013. 01.019

6 Rossi MN and Antonangeli F: LncRNAs: New players in apoptosis control. Int J Cell Biol 2014: 473857, 2014. PMID: 24627686. DOI: $10.1155 / 2014 / 473857$

7 Pickard MR, Mourtada-Maarabouni M and Williams GT: Long non-coding RNA GAS5 regulates apoptosis in prostate cancer cell lines. Biochim Biophys Acta 1832(10): 1613-1623, 2013. PMID: 23676682. DOI: 10.1016/j.bbadis.2013.05.005 
8 Jalali S, Gandhi S and Scaria V: Navigating the dynamic landscape of long noncoding RNA and protein-coding gene annotations in GENCODE. Hum Genomics 10(1): 35, 2016. PMID: 27793185. DOI: 10.1186/s40246-016-0090-2

9 Li P, Zhang D, Su T, Wang W, Yu Y, Zhao X, Li Z, Yu S and Zhang F: Genome-wide analysis of mRNA and lncRNA expression and mitochondrial genome sequencing provide insights into the mechanisms underlying a novel cytoplasmic male sterility system, BVRC-CMS96, in Brassicarapa. Theor Appl Genet 133(7): 2157-2170, 2020. PMID: 32399654. DOI: $10.1007 / \mathrm{s} 00122-020-03587-\mathrm{z}$

10 Gao GD, Liu XY, Lin Y, Liu HF and Zhang GJ: LncRNA CASC9 promotes tumorigenesis by affecting EMT and predicts poor prognosis in esophageal squamous cell cancer. Eur Rev Med Pharmacol Sci 22(2): 422-429, 2018. PMID: 29424900. DOI: 10.26355/eurrev_201801_14191

11 Chang ZW, Jia YX, Zhang WJ, Song LJ, Gao M, Li MJ, Zhao RH, Li J, Zhong YL, Sun QZ and Qin YR: LncRNATUSC7/miR-224 affected chemotherapy resistance of esophageal squamous cell carcinoma by competitively regulating DESC1. J Exp Clin Cancer Res 37(1): 56, 2018. PMID: 29530057. DOI: 10.1186/s13046-018-0724-4

12 Pasic I, Shlien A, Durbin AD, Stavropoulos DJ, Baskin B, Ray PN, Novokmet A and Malkin D: Recurrent focal copy-number changes and loss of heterozygosity implicate two noncoding RNAs and one tumor suppressor gene at chromosome 3q13.31 in osteosarcoma. Cancer Res 70(1): 160-171, 2010. PMID: 20048075. DOI: 10.1158/0008-5472.CAN-09-1902

13 Liu Q, Huang J, Zhou N, Zhang Z, Zhang A, Lu Z, Wu F and Mo YY: LncRNA loc285194 is a p53-regulated tumor suppressor. Nucleic Acids Res 41(9): 4976-4987, 2013. PMID: 23558749. DOI: $10.1093 /$ nar/gkt182

14 Qi P, Xu MD, Shen XH, Ni SJ, Huang D, Tan C, Weng WW, Sheng WQ, Zhou XY and Du X: Reciprocal repression between TUSC7 and miR-23b in gastric cancer. Int J Cancer 137(6): 1269-1278, 2015. PMID: 25765901. DOI: 10.1002/ijc.29516

15 Qiu J, Pan G and Li M: Decreased expression of long noncoding LOC285194 predicts tumour progression and poor prognosis of hepatocellular carcinoma after curative hepatectomy. Postgrad Med J 96(1132): 79-83, 2020. PMID: 31494574. DOI: 10.1136/postgradmedj-2019-136905

16 Ren W, Chen S, Liu G, Wang X, Ye H and Xi Y: TUSC7 acts as a tumor suppressor in colorectal cancer. Am J Transl Res 9(9): 4026-4035, 2017. PMID: 28979678.

17 Yue L and Guo J: LncRNA TUSC7 suppresses pancreatic carcinoma progression by modulating miR-371a-5p expression. J Cell Physiol, 2019. PMID: 30714151. DOI: 10.1002/jcp.28248
18 Yang H, Zhong Y, Xie H, Lai X, Xu M, Nie Y, Liu S and Wan YJ: Induction of the liver cancer-down-regulated long noncoding RNA uc002mbe. 2 mediates trichostatin-induced apoptosis of liver cancer cells. Biochem Pharmacol 85(12): 1761-1769, 2013. PMID: 23643933. DOI: 10.1016/j.bcp.2013.04.020

19 Mehrad-Majd H, Ravanshad S, Moradi A, Khansalar N, Sheikhi $\mathrm{M}$ and Akhtari J: Decreased expression of lncRNA loc285194 as an independent prognostic marker in cancer: A systematic review and meta-analysis. Pathol Res Pract 215(6): 152426, 2019. PMID: 31054796. DOI: 10.1016/j.prp.2019.04.018

20 Sopo M, Anttila M, Muukkonen OT, YlÄ-Herttuala S, Kosma VM, Keski-Nisula L and Sallinen H: Microvessels in epithelial ovarian tumors: High microvessel density is a significant feature of malignant ovarian tumors. Anticancer Res 40(12): 6923-6931, 2020. PMID: 33288586. DOI: 10.21873/anticanres.14716

21 Haigis KM, Cichowski K and Elledge SJ: Tissue-specificity in cancer: The rule, not the exception. Science 363(6432): 11501151, 2019. PMID: 30872507. DOI: 10.1126/science.aaw3472

22 Chou J, Provot S and Werb Z: GATA3 in development and cancer differentiation: cells GATA have it! J Cell Physiol 222(1): 42-49, 2010. PMID: 19798694. DOI: 10.1002/jcp.21943

23 Derrien T, Johnson R, Bussotti G, Tanzer A, Djebali S, Tilgner H, Guernec G, Martin D, Merkel A, Knowles DG, Lagarde J, Veeravalli L, Ruan X, Ruan Y, Lassmann T, Carninci P, Brown JB, Lipovich L, Gonzalez JM, Thomas M, Davis CA, Shiekhattar R, Gingeras TR, Hubbard TJ, Notredame C, Harrow $\mathrm{J}$ and Guigó R: The GENCODE v7 catalog of human long noncoding RNAs: analysis of their gene structure, evolution, and expression. Genome Res 22(9): 1775-1789, 2012. PMID: 22955988. DOI: $10.1101 /$ gr.132159.111

24 Mattioli K, Volders PJ, Gerhardinger C, Lee JC, Maass PG, Melé $\mathrm{M}$ and Rinn JL: High-throughput functional analysis of lncRNA core promoters elucidates rules governing tissue specificity. Genome Res 29(3): 344-355, 2019. PMID: 30683753. DOI: $10.1101 /$ gr.242222.118 